\title{
SECOND COHOMOLOGY AND NILPOTENCY CLASS 2
}

\section{Siniša Crvenković and Vladimir Tasić}

\begin{abstract}
Conditions are given for a class 2 nilpotent group to have no central extensions of class 3. This is related to Betti numbers and to the problem of representing a class 2 nilpotent group as the fundamental group of a smooth projective variety.
\end{abstract}

Surveys of the work on the characterization of the fundamental groups of smooth projective varieties and Kähler manifolds (see $[\mathbf{1}],[\mathbf{3}],[\mathbf{9}]$ ) indicate that torsion-free nilpotent groups have been both attractive and problematic in this context. For example, it took a long time to show, contrary to the beliefs of many researchers in the area, that the fundamental group of a smooth projective variety can be non-abelian torsion-free nilpotent $([\mathbf{2}],[\mathbf{1 1}])$. Even more interestingly from an algebraist's point of view, nilpotent groups of class 2 have played a significant role as test cases. There are structural reasons for this. Let $\mathcal{P}$ denote the class of groups isomorphic to fundamental groups of smooth projective varieties. Let $\gamma_{k}(G)$ denote the $k$-th term of the lower central series of the group $G$. For a subgroup $K$ of $G$, let $\sqrt{K}$ denote the subgroup generated by $\left\{g \in G \mid(\exists n \in \mathbb{Z}) g^{n} \in K\right\}$. Due to the work of Deligne [4], Hain [6] and others, it is known that if $G \in \mathcal{P}$ then the quotients $G / \sqrt{\gamma_{2}(G)}$ and $G / \sqrt{\gamma_{3}(G)}$ in a certain sense determine $G / \sqrt{\gamma_{k}(G)}$ for all $k$. Consequently, if $G \in \mathcal{P}$ is torsion-free nilpotent of class 2 , its class 3 extensions are limited by the underlying geometry.

For this and other reasons, class 2 nilpotent groups have generated a certain amount of interest among geometers. For instance, Hain [7] defines groups of Heisenberg type as finitely generated nontrivial central extensions of $\mathbb{Z}$ by a torsionfree Abelian group, and demonstrates that fundamental groups of links of isolated singularities of $n$-dimensional complex algebraic varieties are of Heisenberg type (hence torsion-free and nilpotent of class 2). The definition generalizes the standard Heisenberg groups $H_{2 m+1}$, given by generators $\left\{x_{0}, x_{1}, \ldots, x_{2 m}\right\}$ and relations $\left(x_{i}, x_{m+i}\right)=x_{0}$ for $i=1, \ldots, m$, and $\left(x_{j}, x_{k}\right)=1$ for all other commutators. These

2000 Mathematics Subject Classification: Primary 20J06, 57T10; Secondary 20F18, 57M05. Key words and phrases: Nilpotent groups, Lie algebras, cohomology, projective varieties.

Research of the first author was supported by the Ministry of Science of the Republic of Serbia, Grant no. 144011. 
groups are well understood, but it is not a trivial matter to show that $H_{2 m+1} \notin \mathcal{P}$. The proof relies on the sophisticated results due to Deligne et al. ([5]; see also [9]) about the degrees of the defining relations of the associated Lie algebra. Despite the powerful algebraic constraints, it is still not known (see [3]) whether the class of Kähler groups, which includes $\mathcal{P}$, contains a nilpotent group of class 3 .

We are interested in a purely algebraic analog of the restriction imposed on class 3 extensions by the underlying geometry in the case of fundamental groups of smooth projective varieties. To this end we introduce the concept of an extension matrix of a finitely generated torsion-free class 2 nilpotent group, and relate it to the second Betti number. The algebraic analog does not capture all restrictions imposed by geometry; for example, Campana [2] has shown that the Lie algebra associated with the fundamental group of a complex projective variety must have dimension at least 9 (which does not follow solely from the algebraic properties of extensions). Nevertheless, taking account of well known properties of Betti numbers of smooth projective varieties, our results can be used to construct new examples of class 2 nilpotent groups that do not belong to $\mathcal{P}$, and to give a new and simpler proof that $H_{4 k+1} \notin \mathcal{P}$.

It is convenient to work with the cohomology of the associated Lie algebra $L(G)$ (over $\mathbb{Q}$ ) constructed in the standard way from the lower central series of $G$. If $G$ is torsion-free and nilpotent of class 2, then $L(G)$ is also nilpotent of class 2, i.e, $L^{3}=[[L, L], L]=0$. Since we are interested in the non-abelian case, we may assume $L^{2}=[L, L] \neq 0$.

If $L$ is a class 2 nilpotent finite-dimensional Lie algebra, choose $X=\left\{x_{1}, \ldots, x_{d}\right\}$ so that $\left\{x_{1}+L^{2}, \ldots, x_{d}+L^{2}\right\}$ is a basis of $L / L^{2}$, and let $Y=\left\{y_{1}, \ldots, y_{e}\right\}$ be a basis of $L^{2}$. Since $L^{3}=0, X \cup Y$ is a basis of $L$, and $L$ is determined by the $\left(\begin{array}{l}d \\ 2\end{array}\right)$ relations of the form:

$$
\left[x_{i}, x_{j}\right]=\sum_{t=1}^{e} \alpha_{i j}^{(t)} y_{t} \quad(i>j)
$$

It is useful to define the structure constants $\alpha_{i j}^{(t)}$ for $i \leqslant j$, by setting $\alpha_{i i}^{(t)}=0$ and $\alpha_{i j}^{(t)}=-\alpha_{j i}^{(t)}$.

Let $\Lambda^{k}(L)$ denote the space of $k$-linear functionals on $L$. Recall that the differential $d_{k}: \Lambda^{k}(L) \rightarrow \Lambda^{k+1}(L)$ is given by

$$
\left(d_{k} f\right)\left(x_{1}, \ldots, x_{k+1}\right)=\sum_{1 \leqslant i<j \leqslant k+1}(-1)^{i+j} f\left(\left[x_{i}, x_{j}\right], x_{1}, \ldots, \hat{x_{i}}, \ldots, \hat{x_{j}}, \ldots, x_{k+1}\right)
$$

for all $f \in \Lambda^{k}(L)$ and $x_{1}, \ldots, x_{k+1} \in L$. The $k$-th cohomology group (which is in fact a vector space) is defined as $H^{k}(L, \mathbb{Q})=\operatorname{ker}\left(d_{k}\right) / \operatorname{Im}\left(d_{k-1}\right)$. Let $b_{k}(L)=$ $\operatorname{dim} H^{k}(L, \mathbb{Q})$, the $k$-th Betti number of $L$. In general, $H^{1}(L, \mathbb{Q})$ is the dual of $L / L^{2}$, so that $b_{1}(L)=\operatorname{dim}\left(L / L^{2}\right)$. We are interested in computing the second Betti number of a Lie algebra of nilpotent class 2, that is, of a non-abelian algebra $L$ for which $L^{3}=0$. For such an $L$, we have $d=b_{1}(L)=\operatorname{dim}\left(L / L^{2}\right)>1$ and $e=\operatorname{dim}\left(L^{2}\right)>0$. 
We assume in the following that $d>2$. This is not a serious restriction. Since $e \leqslant\left(\begin{array}{l}d \\ 2\end{array}\right)$ for all $L, d=2$ implies $e \leqslant 1$. Thus either $e=0$, in which case the algebra is abelian, or $e=1$, in which case $L$ is the three dimensional Heisenberg algebra. The assumption $d>2$ thus excludes only one class 2 nilpotent algebra.

Now consider the vector space $\Lambda^{2}$ of skew-symmetric bilinear functionals on $L$. We must determine the dimension of ker $d_{2}$. The subspace ker $d_{2}$ consists of all the $f \in \Lambda^{2}$ such that for each triple $1 \leqslant k<j<i \leqslant d$ and each pair $1 \leqslant r<t \leqslant e$ the following linear equations hold:

$$
\begin{aligned}
\sum_{t=1}^{e} \alpha_{i j}^{(t)} f\left(y_{t}, x_{k}\right)+\sum_{t=1}^{e} \alpha_{k i}^{(t)} f\left(y_{t}, x_{j}\right)+\sum_{t=1}^{e} \alpha_{j k}^{(t)} f\left(y_{t}, x_{i}\right) & =0 \\
f\left(y_{t}, y_{r}\right) & =0
\end{aligned}
$$

Given $f$, let $f_{t s}=f\left(y_{t}, x_{s}\right)$ and $\vec{f}=\left(f_{11}, \ldots, f_{e 1}, f_{12}, \ldots, f_{e 2}, \ldots, f_{1 d}, \ldots, f_{e d}\right)^{T}$. Then $\vec{f} \in \mathbb{Q}^{e d}$, and the $\left(\begin{array}{l}d \\ 3\end{array}\right)$ equations (1) above can be written as a matrix equation $A \vec{f}=\overrightarrow{0}$ for a suitable matrix $A$ of size $\left(\begin{array}{l}d \\ 3\end{array}\right) \times e d$. We call this matrix the extension matrix of $L$. Its nullity is closely related to class 3 extensions of $L$. It also permits us to compute effectively the second Betti number of $L$.

LEMma 1. Let $L$ be a finite-dimensional class 2 nilpotent Lie algebra with $b_{1}(L) \geqslant 3$ and extension matrix $A$. If $n(A)$ denotes the nullity of $A$, then $b_{2}(L)=$ $\left(\begin{array}{l}d \\ 2\end{array}\right)-e+n(A)$.

Proof. For a functional $f \in \operatorname{ker} d_{2}$, define $T(f)=\vec{f}$ as above. By the earlier discussion we have $T(f) \in \operatorname{ker}(A)$. Thus $T: \operatorname{ker} d_{2} \rightarrow \operatorname{ker}(A)$ is a linear transformation. The kernel of $T$ consists of all functionals such that $f\left(y_{t}, x_{s}\right)=0$ and $f\left(y_{t}, y_{r}\right)=0$ for all $r, t, s$. Since the values of $f\left(x_{i}, x_{j}\right)$ can be assigned arbitrarily for each $i>j$, $\operatorname{dim} \operatorname{ker}(T)=\left(\begin{array}{l}d \\ 2\end{array}\right)$. Given $\vec{f} \in \operatorname{ker}(A)$, define a functional $g \in \operatorname{ker} d_{2}$ by setting $g\left(x_{i}, x_{j}\right)=0, g\left(y_{t}, x_{r}\right)=f_{t r}$ and $g\left(y_{t}, y_{s}\right)=0$. Then $T(g)=\vec{f}$, so $T$ is onto, and we have $\operatorname{dim} \operatorname{Im}(T)=n(A)$. It follows that $\operatorname{dim}\left(\operatorname{ker} d_{2}\right)=\operatorname{dim} \operatorname{ker}(T)+\operatorname{dim} \operatorname{Im}(T)=\left(\begin{array}{l}d \\ 2\end{array}\right)+n(A)$. By definition of $H^{2}(L, \mathbb{Q})$, we have $b_{2}(L)=\operatorname{dim}\left(\operatorname{ker} d_{2}\right)-\operatorname{dim}\left(\operatorname{Im} d_{1}\right)$. But $\operatorname{Im} d_{1}$ is isomorphic to the vector space dual of $L^{2}$, so $\operatorname{dim}\left(\operatorname{Im} d_{1}\right)=e$. Therefore $b_{2}(L)=\left(\begin{array}{l}d \\ 2\end{array}\right)-e+n(A)$.

It is instructive to consider several standard examples. Using Lemma 1, we compute the second Betti numbers of the Heisenberg algebras and of the free class 2 nilpotent algebras.

EXAMPLE 1. The Heisenberg algebra $H_{2 m+1}$ is given by the $2 m+1$ generators $\left\{x_{1}, \ldots, x_{2 m}, y_{1}\right\}$ and relations $\left[x_{i}, x_{m+i}\right]=y_{1}$, with all other brackets equal to zero. Thus we have $d=2 m$ and $e=1$. If $m=1$, there are no relations of type (1) above (hence Lemma 1 does not apply), but it is easy to see that in this case $b_{2}=2$. We assume that $m>1$. Then the extension matrix is of size $\left(\begin{array}{c}2 m \\ 3\end{array}\right) \times 2 m$. 
For example, if $m=2$, the extension matrix is $4 \times 4$ :

$$
A=\left(\begin{array}{cccc}
0 & 0 & -1 & 0 \\
0 & 0 & 0 & 1 \\
1 & 0 & 0 & 0 \\
0 & 1 & 0 & 0
\end{array}\right)
$$

Clearly $n(A)=0$, so by Lemma $1 b_{2}\left(H_{5}\right)=5$. In fact, $n(A)=0$ for all Heisenberg algebras $H_{2 m+1}$. To see this, we note that the structure constants of $H_{2 m+1}$ are such that $\alpha_{i j}=0$ whenever $|i-j| \neq m$ and $\alpha_{i j}=\operatorname{sgn}(i-j)$ if $|i-j|=m$. For each $i, 1 \leqslant i \leqslant d=2 m$, let $\vec{\varepsilon}_{t}$ be the row $t$ of the $d \times d$ identity matrix. We have several cases:

(1) $t<m$. The row of $A$ corresponding to the triple $2 m>m>t$ equals $\vec{\varepsilon}_{t}$.

(2) $2 m>t>m$. The row of $A$ corresponding to this triple equals $-\vec{\varepsilon}_{t}$.

(3) $t=m$. The row corresponding to $m+1>m>1$ equals $-\vec{\varepsilon}_{m}$.

(4) $t=2 m$. The row corresponding to $2 m>m+1>1$ equals $\vec{\varepsilon}_{2 m}$.

Thus $A$ contains a $d \times d$ submatrix of rank $d$. Since $A$ is of size $\left(\begin{array}{l}d \\ 3\end{array}\right) \times d$, it follows that $\operatorname{rank}(A)=d$ and $n(A)=0$. By Lemma $1, b_{2}\left(H_{2 m+1}\right)=\left(\begin{array}{c}2 m \\ 2\end{array}\right)-1$. Note that Santhaourbane $[\mathbf{1 0}]$ has computed the higher Betti numbers of Heisenberg algebras using cohomological methods.

It would be interesting to determine the second Betti numbers for the more general algebras of Heisenberg type defined by Hain $([\mathbf{7}])$. It is not difficult to see that Betti numbers of these more general algebras can be larger than the Betti number of the standard Heisenberg algebra of the same dimension. For example, consider the 5-dimensional algebra $L$ with generators $x_{1}, \ldots, x_{4}, y_{1}$ and relations $\left[x_{4}, x_{1}\right]=y_{1},\left[x_{3}, x_{1}\right]=y_{1}$ and all other brackets zero. Here the extension matrix is

$$
A=\left(\begin{array}{cccc}
0 & 0 & 0 & 0 \\
0 & 0 & 1 & 1 \\
0 & -1 & 0 & 0 \\
0 & -1 & 0 & 0
\end{array}\right)
$$

so $n(A)=2$ and therefore $b_{2}(L)=7$, whereas $b_{2}\left(H_{5}\right)=5$.

EXAMPLE 2. Let $F_{n}$ denote the free class 2 nilpotent Lie algebra on $n \geqslant 3$ free generators. This algebra has a canonical presentation with $d=n$ and $e=\left(\begin{array}{l}n \\ 2\end{array}\right)$ (since there are no relations among the commutators $\left[x_{i}, x_{j}\right], i>j$ ). We want to show that the rank of the extension matrix is maximal, i.e., equal to $\left(\begin{array}{l}d \\ 3\end{array}\right)$. To see this, let $E=\{1, \ldots, e\}$ and let $D_{m}$ be the set of strictly decreasing sequences of length $m$ in $\{1, \ldots, d\} . D_{m}$ and $D_{m} \times E$ can be given a total order, for example the lexicographic order. Thus we can think of rows of the extension matrix $A$ as indexed by $D_{3}$, and its columns indexed by $D_{2} \times E$. With this convention, the upper index of the structure constants $\alpha_{i j}^{(t)}$ ranges over $D_{2}$, and we have $\alpha_{i j}^{(t)}=\operatorname{sgn}(i-j)$ if $t=(i, j)$ or $t=(j, i)$; otherwise $\alpha_{i j}^{(t)}=0$. Now take $(i, j, k) \in D_{3}$. Then $t=(i, j) \in D_{2}$. Consider the column of $A$ indexed by $(t, k)$. The only nonzero entry in this column equals $\alpha_{i j}^{(t)}=1$ and appears in the row indexed by $(i, j, k)$. Thus the extension 
matrix $A$ contains the identity matrix of size $\left(\begin{array}{l}d \\ 3\end{array}\right) \times\left(\begin{array}{l}d \\ 3\end{array}\right)$ as a submatrix, and it follows that the rank of $A$ is $\left(\begin{array}{l}d \\ 3\end{array}\right)$. Therefore $n(A)=d e-\left(\begin{array}{l}d \\ 3\end{array}\right)=d\left(\begin{array}{l}d \\ 2\end{array}\right)-\left(\begin{array}{l}d \\ 3\end{array}\right)=2\left(\begin{array}{c}d+1 \\ 3\end{array}\right)$. Since $e=\left(\begin{array}{l}d \\ 2\end{array}\right)$, by Lemma 1 we have $b_{2}\left(F_{n}\right)=2\left(\begin{array}{c}n+1 \\ 3\end{array}\right)$.

Turning back to extensions of class 3 , it is well known that the elements of $H^{2}(L)$ are the equivalence classes of central extensions (see [8]). However, some of the extensions have nilpotent class 2 . Let $C_{2} \subseteq H^{2}(L)$ denote the set of equivalence classes of class 2 extensions of $L$. It is not difficult to see (and it is demonstrated in the proof of the lemma below) that $C_{2}$ is a subspace of $H^{2}(L)$. Lemma 2 establishes the dimension of the space $H^{2} / C_{2}$ and so in a sense 'measures the number' of class 3 extensions.

Lemma 2. Let $L$ be a finite-dimensional class 2 nilpotent Lie algebra with $b_{1}(L) \geqslant 3$ and the extension matrix $A$. Then $H^{2}(L) / C_{2} \cong \operatorname{ker}(A)$. Thus, modulo class 2 extensions, $L$ has precisely $n(A)$ basic extensions of class 3.

Proof. Each functional $f \in \operatorname{ker} d_{2}$ defines a central extension $L_{1}$ on the vector space $L \oplus \mathbb{Q}$. The bracket operation on $L_{1}$ is defined by:

$$
\begin{aligned}
& {[u, q]_{1}=0} \\
& {[u, v]_{1}=[u, v]+f(u, v)}
\end{aligned}
$$

for $u, v \in L$ and $q \in \mathbb{Q}$. Since the elements of $\mathbb{Q}$ are central in $L_{1}$, it is clear that $L_{1}$ is of class 2 if and only if $\left[x_{s}, y_{t}\right]_{1}=0$ for all $s$ and $t$. But $L$ is of class 2, so $\left[x_{s}, y_{t}\right]=0$ and hence $\left[x_{s}, y_{t}\right]_{1}=\left[x_{s}, y_{t}\right]+f\left(x_{s}, y_{t}\right)=f\left(x_{s}, y_{t}\right)$. Thus $L_{1}$ is of class 2 if and only if $f\left(x_{s}, y_{t}\right)=0$ for all $s$ and $t$. Therefore $f \in \operatorname{ker} d_{2}$ defines a class 2 extension if and only if $f \in \operatorname{ker}(T)$, where $T: \operatorname{ker} d_{2} \rightarrow \operatorname{ker}(A)$ is the linear transformation defined in Lemma 1 by $T(f)=\vec{f}$. Since the set of functionals defining extensions of class 2 is $\operatorname{ker}(T)$, we have $C_{2}=\left(\operatorname{ker}(T)+\operatorname{Im}\left(d_{1}\right)\right) / \operatorname{Im}\left(d_{1}\right)$. But $\operatorname{Im}\left(d_{1}\right) \subseteq \operatorname{ker}(T)$. To see this, suppose $f=d_{1} g$ for some linear functional $g: L \rightarrow \mathbb{Q}$. Then $f\left(x_{s}, y_{t}\right)=d_{1} g\left(x_{s}, y_{t}\right)=-g\left(\left[x_{s}, y_{t}\right]\right)=-g(0)=0$ because $L$ is of nilpotent class 2 and $y_{t} \in L^{2}$. Therefore $C_{2}=\operatorname{ker}(T) / \operatorname{Im}\left(d_{1}\right)$, and we have

$$
H^{2}(L) / C_{2} \cong\left(\operatorname{ker} d_{2} / \operatorname{Im}\left(d_{1}\right)\right) /\left(\operatorname{ker}(T) / \operatorname{Im}\left(d_{1}\right)\right) \cong \operatorname{ker} d_{2} / \operatorname{ker}(T) .
$$

By an argument given in the proof of Lemma $1, T: \operatorname{ker} d_{2} \rightarrow \operatorname{ker}(A)$ is surjective, so $\operatorname{ker} d_{2} / \operatorname{ker}(T) \cong \operatorname{ker}(A)$. Hence $H^{2}(L) / C_{2} \cong \operatorname{ker}(A)$.

The lemmas imply:

THEOREM 1. Let $L$ be a finite-dimensional class 2 nilpotent Lie algebra with the extension matrix $A$ and $b_{1}(L) \geqslant 3$. Then $L$ has no central extensions of nilpotent class 3 if and only if $n(A)=0$.

EXAMPLE 3. Some Lie algebras (and groups) of Heisenberg type admit central extensions of class 3 . Let $L$ be the algebra generated by $\left\{x_{1}, \ldots, x_{6}, y_{1}\right\}$, with relations $\left[x_{6}, x_{5}\right]=y_{1},\left[x_{5}, x_{4}\right]=y_{1}$ and all other brackets zero. The extension matrix $A$ is of size $20 \times 6$ and has nullity 2. Recall from Example 2 that rows of $A$ are indexed by strictly decreasing sequences of length 3 from $\{1, \ldots 6\}$; we assume 
the sequences are given the reverse lexicographic order. With this convention, the submatrix consisting of the first four rows of $A$ is

$$
\left(\begin{array}{llllll}
0 & 0 & 0 & 1 & 0 & 1 \\
0 & 0 & 1 & 0 & 0 & 0 \\
0 & 1 & 0 & 0 & 0 & 0 \\
1 & 0 & 0 & 0 & 0 & 0
\end{array}\right)
$$

The only other nonzero rows are those indexed by $(5,4,3),(5,4,2)$ and $(5,4,1)$. However, these rows have zeros in columns 5, 6 and 7 , so the nonzero entries can be eliminated by using rows 2,3 and 4 of $A$. Hence $n(A)=2$.

It is interesting to formulate Theorem 3 without referring to the extension matrix, that is, in terms of the second Betti number.

THEOREM 2. Let $L$ be a finite-dimensional class 2 nilpotent Lie algebra such that $b_{1}(L) \geqslant 3$. Then

(1) $b_{2}(L) \geqslant\left(\begin{array}{l}d \\ 2\end{array}\right)-e$

(2) $L$ does not admit central extensions of class 3 if and only if $b_{2}(L)=\left(\begin{array}{l}d \\ 2\end{array}\right)-e$.

The condition $n(A)=0$, given in Theorem 3 , forces restrictions on the parameters $d$ and $e$ that are not explicit in Theorem 4. Since the extension matrix $A$ is of size $\left(\begin{array}{l}d \\ 3\end{array}\right) \times e d$, its null space will be of positive dimension if $e d>\left(\begin{array}{l}d \\ 3\end{array}\right)$. Thus if $L$ has no central extensions of class 3 , then $e d \leqslant\left(\begin{array}{l}d \\ 3\end{array}\right)$. An elementary computation now yields:

COROLlary 1. Let $L$ be a class 2 nilpotent finite-dimensional Lie algebra such that $b_{1}(L) \geqslant 3$. If $L$ has no central extensions of class 3 , then $b_{2}(L)=\left(\begin{array}{l}d \\ 2\end{array}\right)-e$ and $e \leqslant \frac{(d-1)(d-2)}{6}$. In particular, $b_{2}(L) \geqslant \frac{d^{2}-1}{3}$.

ExAmple 4. Suppose $L$ is a 4-dimensional class 2 nilpotent algebra that has no extensions of class 3 . Then $\operatorname{dim}(L)=d+e=4$, so either $d=2$ and $e=2$ or $d=3$ and $e=1$. ( $e=0$ is impossible because then $L$ is abelian, and $d=1$ is impossible because then $L$ is abelian and $e=0$.) But if $d=2$ then $e \leqslant 1$, so $d+e<4$. Therefore $d=3$ and $e=1$, which contradicts Corollary 5. Hence every 4-dimensional class 2 algebra admits an extension of class 3.

To formulate the group analog to Corollary 5 , note that if $G$ is a finitely generated torsion-free class 2 nilpotent group, then $G / G^{\prime}$ and $G^{\prime}$ are finitely generated torsion-free abelian groups. Thus $G$ can be given a 'canonical' presentation analogous to the one we have been using for finite-dimensional class 2 Lie algebras (with structure constants in $\mathbb{Z}$ ). Thus we can speak of the extension matrix of $G$, meaning the extension matrix of the associated Lie algebra.

Corollary 2. Let $G$ be a torsion-free nilpotent group of class 2 , given by its canonical class-2 presentation with $b_{1}(G) \geqslant 3$. If $G$ has no central extensions of class 3 , then $b_{2}(G)=\left(\begin{array}{l}d \\ 2\end{array}\right)-e$ and $e \leqslant \frac{(d-1)(d-2)}{6}$. In particular, $b_{2}(G) \geqslant \frac{d^{2}-1}{3}$.

Both corollaries give only necessary conditions for an algebra or a group not to have central extensions of class 3 . The necessary and sufficient condition is given by 
$n(A)=0$, and must be decided based on the concrete presentation. Nevertheless, the results presented here, together with known restrictions on the cohomology of smooth varieties, imply that certain class 2 nilpotent groups (including the Heisenberg groups $H_{4 m+1}$ ) are not isomorphic to the fundamental group of any smooth variety.

Before we formulate the statement, we note a well-known result due to Deligne (see [4]): if $G \in \mathcal{P}$, the cohomology groups of the associated Lie algebra have a mixed Hodge structure. Therefore Betti numbers $b_{2 n+1}(L)$ are even for all $n$. In particular, $d=b_{1}(L)$ is even, so either $b_{1}(L) \equiv 0(\bmod 4)$ or $b_{1}(L) \equiv 2(\bmod 4)$.

Corollary 3. Let $G$ be a torsion-free class 2 nilpotent group with extension matrix $A$ and $b_{1}(G) \geqslant 3$.

(1) Suppose $b_{1}(G) \equiv 0(\bmod 4)$. If $e$ is odd and $n(A)$ is even, then $G \notin \mathcal{P}$.

(2) Suppose $b_{1}(G) \equiv 2(\bmod 4)$. If e is odd and $n(A)$ is odd, then $G \notin \mathcal{P}$.

Proof. To prove part (1), suppose $G \in \mathcal{P}$. Let $L=L(G)$ be the Lie algebra associated with $G$. Since $G \in \mathcal{P}$, odd Betti numbers of $L$ are even. By Poincaré duality, $b_{2}(L)=b_{d+e-2}(L)$. Since $d+e$ is odd, so is $d+e-2$, and it follows that $b_{2}(L)=b_{d+e-2}(L)$ is even. By Lemma 1 we have $b_{2}(L)=\left(\begin{array}{l}d \\ 2\end{array}\right)-e+n(A)$. But $\left(\begin{array}{l}d \\ 2\end{array}\right)$ is even because $d$ is divisible by 4 , so $n(A)-e \equiv b_{2}(L) \equiv 0(\bmod 2)$. Hence $n(A) \equiv e$ (mod 2), which contradicts the assumption. Thus $G \notin \mathcal{P}$.

The proof of part (2) is very similar. Suppose $G \in \mathcal{P}$. Since $d+e$ is odd, $b_{2}(L)=\left(\begin{array}{l}d \\ 2\end{array}\right)-e+n(A)=b_{d+e-2}(L) \equiv 0(\bmod 2)$, as above. But now $\left(\begin{array}{l}d \\ 2\end{array}\right), e$ and $n(A)$ are each odd, so $b_{2}(G)=\left(\begin{array}{l}d \\ 2\end{array}\right)-e+n(A) \equiv 1(\bmod 2)$. Therefore $G \notin \mathcal{P}$.

The corollary excludes the Heisenberg groups $H_{4 k+1}$ from $\mathcal{P}$, because for these groups $d=4 k, e=1$ and $n(A)=0$. However, Corollary 7 also applies to some groups of Heisenberg type which do have central extensions of class 3.

EXAMPLE 5 . Let $G$ be the group generated by $\left\{x_{1}, \ldots, x_{8}, y_{1}\right\}$, with relations $\left(x_{8}, x_{7}\right)=\left(x_{7}, x_{6}\right)=y_{1}$ and all other commutators are identity. By an argument similar to the one given in Example 3, the extension matrix has nullity 2. Since $d=8$ and $e=1$, Corollary 7 implies $G \notin \mathcal{P}$.

\section{References}

[1] D. Arapura, Fundamental Groups of Smooth Projective Varieties, Complex Algebraic Geometry, MSRI Publications, Vol. 28 (1995).

[2] F. Campana, Remarques sur les groupes de Kähler nilpotents, Ann. Sci. École Norm. Sup. (4) 28:3 (1995), 307-316.

[3] F. Campana, T. Peternell, Recent Developments in the Classification Theory of Kähler Manifolds, Several Complex Variables, MSRI Publications, Vol. 37 (1999).

[4] P. Deligne, Théorie de Hodge, I, Actes du Congrès International des Mathématiciens, I, Nice (1970), 425-430.

[5] P. Deligne, P. Griffiths, J. Morgan, and D. Sullivan, Real homotopy theory of Kähler manifolds, Invent. Math. 29:3 (1975), 245-274.

[6] R. N. Hain, The Geometry of the Mixed Hodge Structure on the Fundamental Group; in: 'Algebraic Geomtery Bowdoin', Proc. Symp. Pure Mathematics, AMS, Rhode Island, 1986. 
[7] R. N. Hain, Nil-manifolds as links of isolated singularities, Compositio Math. 84:1 (1992), 91-99.

[8] P. J. Hilton, U. Stambach, A Course in Homological Algebra, Springer-Verlag, New York, 1971.

[9] F. Johnson and E. Rees, The Fundamental Group of an Algebraic Variety, Lecture Notes in Mathematics 1474, Springer-Verlag, New York, 1991.

[10] L. J. Santharoubane, Cohomology of Heisenberg Lie Algebras, Proc. Am. Math. Soc. 87:1 (1983), 23-28.

[11] A. Sommese and A. van de Ven, Homotopy groups of pullbacks of varieties, Nagoya Math. J. 102 (1986), 79-90.

Prirodno-matematički fakultet

21000 Novi Sad

Serbia

sima@eunet.yu

Department of Mathematics and Statistics

University of New Brunswick

Fredericton, NB

Canada E3B 5A3

vtasic@unb.ca 